\title{
Strates
}

STRATES Matériaux pour la recherche en sciences sociales

15 | 2008

Union européenne - Voisinages. La quête d'une intégration régionale

\section{L'Union européenne et ses périphéries : faisabilité du scénario des dominos}

Jean-François Drevet

\section{OpenEdition}

Journals

Édition électronique

URL : http://journals.openedition.org/strates/6526

DOI : $10.4000 /$ strates.6526

ISSN : $1777-5442$

Éditeur

Laboratoire Ladyss

Édition imprimée

Date de publication : 1 janvier 2008

Pagination : 25-36

ISSN : 0768-8067

Référence électronique

Jean-François Drevet, «L'Union européenne et ses périphéries : faisabilité du scénario des dominos », Strates [En ligne], 15 | 2008, mis en ligne le 04 mars 2013, consulté le 08 septembre 2020. URL : http:// journals.openedition.org/strates/6526; DOI : https://doi.org/10.4000/strates.6526

Ce document a été généré automatiquement le 8 septembre 2020.

Tous droits réservés 


\title{
L'Union européenne et ses périphéries : faisabilité du scénario des dominos
}

\author{
Jean-François Drevet
}

\section{NOTE DE L'ÉDITEUR}

Les opinions exprimées ici n'engagent que l'auteur.

1 Depuis le $1^{\text {er }}$ janvier 2007, l'Union européenne (l'UE) compte 27 États. Bien qu'en moins de deux décennies elle ait plus que doublé le nombre de ses membres, son élargissement n'est pas achevé. À la Croatie et à la Turquie déjà engagées dans des négociations d'adhésion, s'ajoutent les autres pays des Balkans et d'autres pays de la périphérie orientale et méridionale, qui sont candidats à la candidature et attendent une occasion favorable pour déposer officiellement leur demande.

2 Qu'ils s'inscrivent ou non dans une perspective d'adhésion, tous ces pays sont engagés dans un mouvement de recomposition qui n'a qu'un mot d'ordre : no western borders. Une vingtaine de peuples se rapproche du seul ensemble qui puisse leur offrir, soit une perspective d'intégration, soit un processus de rapprochement graduel dans le cadre de la nouvelle politique européenne de voisinage (PEV).

3 Est-il possible de maîtriser cette évolution, parfois qualifiée de scénario des dominos, qui n'est actuellement limitée, ni dans le temps, ni dans l'espace? Dans ce vaste problème, plusieurs aspects stratégiques sont à prendre en considération :

4 - le rôle des critères politiques dans la dynamique d'intégration ;

5 - l'agrandissement de l'UE n'a-t-il pas de limite?

6 - l'influence des forces contraires ;

7 - l'impact possible de la PEV. 


\section{Le rôle des critères politiques}

8 Après avoir accepté une moyenne de trois nouveaux pays par décennie (1973-1995), les adhésions de 2004-2007 montrent que l'élargissement n'est plus un événement mais un processus. Dans la nouvelle file d'attente qui s'est constituée, les problèmes surpassent ceux des adhérents antérieurs: leurs économies, notamment celles qui se sont récemment dégagées de la planification centralisée, rencontrent des difficultés énormes et leur pratique de la démocratie est souvent sujette à caution.

Or, depuis les origines de l'Union et bien qu'elle n'ait longtemps été qu'un marché commun, l'exigence démocratique est un critère de base de l'adhésion. Pour cette raison, l'Espagne franquiste n'a pu signer autre chose qu'un accord commercial avec la CEE et les accords d'association de 1963 ont été suspendus à la suite des coups d'État militaires en Grèce (1967) et en Turquie (1980).

10 Au début des années 1990, face à une vague de candidatures de pays récemment démocratisés, l'UE a décidé d'être plus explicite en citant l'impératif démocratique parmi les critères de Copenhague ${ }^{1}$, puis en l'inscrivant dans les bases juridiques $d u$ traité d'Amsterdam². En 1997, en demandant aux candidats de ne pas «importer de conflits frontaliers dans l'UE $»^{3}$, la Commission a élargi les critères de Copenhague aux relations internationales. Ainsi les candidats d'Europe centrale ont-ils été contraints de signer des accords de coopération et de bon voisinage, qui ont stabilisé cette partie du continent en enterrant les vieux conflits.

11 Pour évaluer la conformité des systèmes politiques des candidats aux normes en vigueur dans l'UE, la Commission a établi une batterie de conditions, concernant à la fois l'état de la démocratie dans le pays candidat (critères internes) et de ses relations internationales (critères externes). Dans ses rapports annuels, elle formule des appréciations très détaillées, aussi bien sur le cadre législatif que les pratiques en vigueur. Ces critères ont donc beaucoup de chances de représenter un des aspects fondamentaux du processus d'intégration, sinon un préalable à l'ouverture de négociations d'adhésion. Ils sont également un facteur clé dans la gestion des accords d'association, de partenariat et de voisinage.

12 L'agrandissement de l'UE à 27 États membres correspond donc à l'extension de la démocratie sur le continent, d'abord au sud dans les années 1970, puis à l'est dans la décennie 1990. Cette évolution est-elle appelée à continuer ?

13 Après la grande phase de négociations qui a conduit une douzaine de candidats vers l'adhésion en 2004 et 2007, celle qui est en cours avec deux pays paraît moins spectaculaire. En fait, elle est d'une dimension similaire : la Croatie est à l'avant-garde d'un groupe de candidats déclarés (la Macédoine) ou potentiels (les autres républiques ex-yougoslaves et l'Albanie) et la Turquie à elle seule représente une population comparable à celle des dix adhérents de 2004.

14 La Croatie mise à part, la réalisation de la promesse d'adhésion des pays balkaniques se heurte à de nombreux problèmes économiques et politiques. En clair, plusieurs de ces pays n'ont pas encore la capacité d'assumer les obligations d'un État membre de l'Union. Après avoir accueilli des États récemment constitués (les Baltes, la Slovénie, la Slovaquie), l'UE est face à des États en construction, dont elle exerce une partie des compétences, dans le cadre des opérations de rétablissement de la paix entreprises avec le concours des Nations unies. Plus de dix années après la signature des accords de 
Dayton, dans l'attente de l'indépendance du Kosovo, elle doit gérer en parallèle la formation de nouveaux États et leur rapprochement avec l'UE.

Ces critères politiques sont également le principal obstacle à l'adhésion de la Turquie, qui doit à la fois poursuivre ses progrès vers un plus grand respect des droits de l'homme et des minorités à l'intérieur et une amélioration de ses relations de voisinage. La liste des dispositions à respecter est longue et concerne un nombre important de sujets très sensibles : le rôle de l'armée, le problème kurde, les relations avec la Grèce et Chypre $^{4}$. En refusant de reconnaître ce dernier pays, État membre de l'UE depuis 2004, le gouvernement d'Ankara s'est engagé dans une impasse, un pays candidat ne pouvant adhérer à ses propres conditions. En outre, la négociation d'adhésion est intergouvernementale: l'ouverture et la clôture de chacun de ses 35 chapitres sont subordonnées à un accord unanime, ce qui permet à un seul pays de tout bloquer.

Concernant les candidats à la candidature, les critères politiques correspondent à des exigences très élevées par rapport à leur situation actuelle. La plupart des pays de la mer Noire ou de la Méditerranée sont loin d'y satisfaire et pas nécessairement désireux de le faire. Leurs dirigeants, qui ne voient déjà pas d'un bon œil les remontrances exprimées par la Commission dans le cadre des accords d'association ou de partenariat en vigueur, n'ont pour le moment aucune envie de s'astreindre aux obligations bien plus sensibles qui s'appliquent aux pays candidats.

Mais cette situation peut évoluer : autour de la mer Noire et de la Méditerranée, le cheminement vers la démocratie se poursuivra. Si les démocraties sont fragiles, les régimes autoritaires le sont également: les événements inattendus n'ont pas manqué en Europe depuis l'ouverture du mur de Berlin.

Dans les républiques ex-soviétiques, l'effondrement du système Chevardnadzé en Géorgie à la fin de 2003, puis les premières élections libres en Ukraine ont semblé donner un signal positif. Si la propagation de la démocratie telle qu'observée en Europe centrale en 1989 ne s'est pas reproduite, les gouvernements doivent de plus en plus souvent composer avec les aspirations des peuples.

Dans les pays du sud et de l'est méditerranéen (PSEM), la situation est moins encourageante. Les systèmes autocratiques qui se sont imposés depuis les indépendances sont toujours en place. La contestation la plus virulente vient de partis islamistes qui veulent établir des gouvernements tout aussi autoritaires. Le processus électoral lui-même est perçu avec méfiance, dans la mesure où il peut accélérer leur arrivée au pouvoir.

20 Tous ces pays étant orientés vers l'économie de marché et pas nécessairement plus difficiles à gérer que les candidats déclarés, il est clair que le processus démocratique est le point crucial d'une éventuelle démarche d'adhésion: pas de démocratie, pas d'adhésion, ce qui laisse un peu de temps à l'Union pour mieux définir sa politique.

21 Mais cela joue aussi dans l'autre sens. Depuis les années 1970, à la suite de l'effondrement du régime des colonels en Grèce, il a été implicitement admis que tout État européen venu ou revenu à la démocratie avait vocation à rejoindre l'UE. Dans les années 1990, le précédent méditerranéen a été invoqué avec succès par les pays d'Europe centrale et il est aujourd'hui soulevé à nouveau par l'Ukraine et la Géorgie : «si nous devenons pleinement démocratiques, vous devez nous offrir une perspective d'intégration » ont dit et répété les responsables de ces pays, ce qui peut mener l'Union très loin vers l'est et le sud. 


\section{L'élargissement, jusqu'où?}

Depuis les adhésions de 2004, un débat s'est engagé sur les limites de l'UE. Dans un rapport de mars 2006, le Parlement européen a souhaité qu'on définisse des frontières géographiques. Ces discussions interviennent dans le contexte d'une interrogation de l'opinion, à la recherche de déterminants géographiques, linguistiques ou culturels, éventuellement religieux, d'un ensemble européen qui n'a jamais été clairement délimité.

Dans la pratique, chaque État membre décide de la composition du territoire sous sa souveraineté à inclure dans l'UE. À plusieurs reprises, il l'a fait en incluant des territoires non européens : les quatre départements français d'outre mer font partie de $l^{1} \mathrm{UE}^{5}$, comme les presidios espagnols ${ }^{6}$ de la côte marocaine. Pour leur part, les autorités britanniques ont inclus Gibraltar, mais ni l'île de Man et l'archipel anglo-normand, ni leurs bases de souveraineté à Chypre et leurs possessions d'outre mer? .

C'est pourquoi, dans un rapport au Conseil de Lisbonne, en 1992, la Commission a estimé que "le terme d'Européen ne pouvant être officiellement défini », qu'il n'était «ni possible, ni opportun d'établir les frontières de l'UE, dont les contours seront encore redéfinis dans les années à venir ».

5 En fait, ni les frontières actuelles de l'UE, ni leur extension aux limites sud et est de la Turquie ne comportent de cohérence géographique. Indépendamment de ses mérites propres, la candidature turque est porteuse d'un effet géopolitique de contagion à un ensemble de pays de la mer Noire, du Proche Orient et de la Méditerranée. Comment dire aux Arméniens ou aux Géorgiens qu'ils seraient moins européens que les Turcs ? La Transcaucasie peut-elle être durablement exclue, si l'Asie mineure se prépare à une intégration pleine et entière? Certes, le Bosphore est moins large que le détroit de Gibraltar : mais si l'EU traverse le premier, comment ne pas penser qu'elle pourrait un jour franchir le second?

Ex-républiques soviétiques, les pays baltes sont maintenant des États membres de plein droit. Grâce à leurs efforts et au soutien sans faille des pays nordiques, l'adhésion leur a été offerte en compensation de la perte de leur indépendance après la Deuxième Guerre mondiale. Bien qu'elle soit également victime du pacte Molotov-Ribbentrop, la Moldavie n'a pas eu cette chance. Mais elle ne considère pas sa frontière avec la Roumanie comme une limite définitive de l'UE et aurait bien voulu être admise à signer comme les Balkaniques un pacte de stabilisation et d'association. Pour rejoindre l'UE, le gouvernement de Chisinau n'aurait-il d'autre choix que de rechercher une union avec la Roumanie?

Depuis la fin des années 1980, les départements d'outre mer (DOM) français, les Canaries, les Açores et Madère forment un groupe de régions tellement ultrapériphériques qu'il s'étend bien au-delà des limites de l'Europe, même très largement considérées. Faut-il s'étonner que l'archipel du Cap Vert, appartenant comme les îles espagnoles et portugaises de l'Atlantique à la Macaronésie ${ }^{8}$, mais État indépendant membre du groupe ACP, y trouve un précédent et s'interroge sur un possible destin européen? 


\section{Les forces contraires}

Il n'est nullement acquis que le scénario des dominos soit la référence tendancielle dans l'expansion de l'Union. La vague de fond qui s'est levée en 1989, en ouvrant à l'UE une aire d'expansion apparemment illimitée à l'est, n'est pas irrésistible et pourrait s'arrêter aux limites actuelles si elle se heurte à des forces contraires. Trois retournements majeurs peuvent contrarier l'évolution décrite plus haut, dans un contexte de montée des tensions aux périphéries est et sud de l'Union, qui ferait reculer son influence : une nouvelle marche en avant de la Russie, une réorientation de la Turquie vers l'est et l'émergence de régimes islamistes ou équivalents dans les PSEM.

Le scénario des dominos suppose une assez grande passivité de la Russie, qui n'aurait pas d'autre choix que d'accepter l'intégration graduelle de quatre membres de la $\mathrm{CEI}^{9}$ (l'Ukraine, la Moldavie, la Géorgie et l'Arménie) qui en ont plus ou moins vigoureusement exprimé le désir. Actuellement, beaucoup de signaux vont dans l'autre sens. Après avoir tenté de les retenir par la force militaire, Moscou utilise aujourd'hui des méthodes plus efficaces (chantage à l'interruption des fournitures d'énergie, action sur les minorités russes, prise de contrôle d'infrastructures stratégiques), qui sont de nature à faire réfléchir n'importe quel gouvernement, même devenu démocratique.

ende étape serait de reprendre l'offensive vers des pays affaiblis en leur imposant des traités inégaux qui les replaceraient sous la dépendance d'un ensemble politique grand russe. La réunification de la Russie d'Europe n'est pas une hypothèse invraisemblable, avec la création d'une Union slave, à laquelle la Biélorussie est déjà prête à adhérer, qui s'étendrait à l'Ukraine, pour autant qu'elle soit prête à devenir une grande Catalogne et même au Kazakhstan, où les Russes sont particulièrement nombreux. Pour le moment, on ne voit pas de pays non slaves, comme la Moldavie ou la Géorgie renoncer à leur souveraineté. Mais une pression très forte de Moscou dans le sens d'une réincorporation de ces pays dans une fédération fortement structurée fait partie des possibilités.

31 Également soumise à des pressions énergétiques, divisée entre partisans de la fermeté et de la souplesse, l'Union ne serait pas en mesure de s'y opposer, en dépit des efforts des nouveaux membres pour muscler la politique européenne vis-à-vis de Moscou. Depuis 1958, l'Europe s'arrête où commence la zone d'influence de la Russie. La limite aurait simplement été reculée vers l'est pour reprendre un tracé plus ancien au-delà de la Pologne et de la Roumanie, à moins que la Moldavie ou une partie de la Transcaucasie ne parviennent à échapper à l'emprise de Moscou.

Si elle affirmait sa volonté de ne pas satisfaire aux critères politiques de l'adhésion ou s'estimait rejetée par l'UE, la Turquie renoncerait à sa candidature. Le nationalisme ombrageux d'Ankara rend cette hypothèse concevable, sinon souhaitable pour les Européens qui n'en veulent pas, même si elle satisfait aux critères de l'adhésion. Si elle n'a probablement pas d'autre choix économique que de rester dans l'union douanière, elle pourrait tenter de construire une autre communauté avec des pays musulmans et turcophones du Moyen Orient et de l'Asie centrale. Revigoré par la dislocation de l'URSS, le rêve du panturquisme, périodiquement agité par les cercles nationalistes comme alternative à l'intégration européenne, reviendrait à l'ordre du jour. Il s'agirait avant tout d'un a priori politique, pas d'une préférence entre deux modes de développement, car les Turkestans n'offrent pas un marché aussi important que l'UE. Des complémentarités appréciables existent cependant, notamment en matière 
énergétique. La Turquie pourrait se procurer plus facilement le gaz et le pétrole qui lui font défaut et trouver un marché pour ses produits agricoles et industriels qui n'intéressent pas le marché européen. Dans une zone fragile, ce scénario implique aussi des tensions entre la Turquie et la Russie ou même l'Iran, qui ne manqueraient pas d'avoir des effets plus larges, y compris sur l'Europe. Dans cette hypothèse, l'Europe garderait pour frontière les limites du traité de Lausanne (1923), sauf à Chypre, où le fait accompli de l'invasion de1974 resterait un abcès de fixation.

Enfin, voici presque deux décennies qu'on redoute l'émergence de régimes xénophobes sur la rive sud de la Méditerranée. Le processus déjà bien insuffisant de coopération entre les deux rives serait compromis par des surenchères violentes, sinon des conflits. Sans même penser au pire, un simple refroidissement aurait pour l'Europe et notamment pour ses régions du sud des conséquences très dommageables. La médiane qui partage le bassin méditerranéen serait confirmée comme une zone de rupture entre l'Europe et l'Afrique.

34 L'Europe, qui n'est plus une forteresse assiégée depuis le XVII ${ }^{e}$ siècle et a pu étendre son influence bien au-delà de ses limites géographiques, a le plus grand intérêt à ne pas donner prise à de tels scénarios. Tout en n'étant pas maîtresse de l'évolution de ses voisins, elle dispose d'une marge d'action qui doit éviter aux relations avec sa périphérie de basculer dans une logique de confrontation.

\section{Vers un dépassement du problème : la politique européenne de voisinage}

Lancée en 2003, la politique européenne de voisinage (PEV) est un produit méthodologique de la dynamique de l'élargissement: elle a été mise au point par des fonctionnaires qui venaient de passer sept années (1995-2002) à préparer les pays candidats à l'adhésion. Comme l'avait indiqué le président Prodi («tout sauf les institutions »), elle couvre la plus grande partie des domaines d'action communautaire (la majorité des 31 chapitres des négociations de 1998-2003, devenus aujourd'hui 35). En principe, elle concerne 16 pays $^{10}$, la Russie bénéficiant d'un partenariat stratégique spécial, mais émargeant au budget de la PEV.

Mais elle doit être mise en œuvre dans le cadre beaucoup plus restreint des relations bilatérales avec les pays Meda ${ }^{11}$ et Tacis ${ }^{12}$, qui ont une expérience de coopération à la carte avec l'UE dans des domaines relativement limités, centrés sur les relations commerciales, notamment les échanges agricoles et l'assistance financière. Bien que le processus de Barcelone ait déjà élargi ce champ d'action dans les PSEM, les réalisations n'ont pas suivi et la coopération est souvent restée au niveau des objectifs.

Les communications de la Commission ont délimité un vaste champ de coopération qui a progressivement été exploré dans la préparation des plans d'action qui doivent servir de cadre aux interventions.

La Commission a été plus loin que dans les accords antérieurs en offrant une possibilité d'association au marché unique, à partir d'une extension progressive des quatre libertés ${ }^{13}$. Compte tenu de l'élargissement de l'éventail des politiques communautaires au cours des dix dernières années, c'est un très vaste champ d'action qui s'ouvre aux pays bénéficiaires de la PEV. Dans la réalité, les opportunités sont plus limitées et souvent assorties de fortes contraintes. 

de l'importance du risque migratoire, qu'il émane des pays d'émigration (les PSEM à l'exception d'Israël) ou des pays de transit (les pays d'Europe orientale et les PSEM, qui reçoivent de plus en plus d'immigrants d'Afrique noire). Pour mieux contrôler ses frontières externes, l'UE cherche à conclure des accords de réadmission avec ses voisins, ce qui revient à reporter la charge de l'immigration clandestine sur les pays de départ (ce qui est logique) mais aussi les pays de transit (eux-mêmes victimes du phénomène). Face à ces obligations, elle offre peu de contreparties : la politique de LSJ (Liberté, Sécurité Justice, ex-JAI) a encore une forte dimension intergouvernementale ce qui la rend très peu attractive $e^{14}$, notamment pour les PSEM. Les frontières occidentales de l'Ukraine aujourd'hui, de la Moldavie demain, sont affectées par l'extension de l'espace Schengen aux nouveaux États membres. Cependant, depuis la fin de 2006, la possibilité d'un donnant donnant en matière migratoire offre des possibilités de progrès vers une gestion concertée des flux : comme cela a été fait avec l'Ukraine, les pays qui lutteront efficacement contre l'immigration clandestine et accepteront les contraintes des accords de réadmission seront récompensés par une attribution plus libérale de visas. Mais il s'agirait plutôt d'une politique de fermeture concertée que de la mise en œuvre de la libre circulation des personnes. d'association. Il figure dans les accords de partenariat et d'association avec les républiques ex-soviétiques. Il était envisagé pour 2010 avec les PSEM, dans le cadre du processus de Barcelone. Les nouvelles propositions de la Commission, sur le modèle des facilités accordées aux pays d'Europe centrale au début des années 1990, puis aux Balkans occidentaux, vont plus loin en offrant aussi la réduction des barrières agricoles. Car la logique du système actuel est de cultiver la tomate sous abri plastique dans le sud de l'Europe avec des aides communautaires et de la main-d'œuvre immigrée du Maghreb, au lieu de laisser le Maroc profiter de ses avantages comparatifs pour les produire chez lui.

41 Les PSEM, qui bataillent depuis les années 1970 pour améliorer leur accès au marché communautaire et n'ont bénéficié que d'ouvertures chichement mesurées, vont-ils obtenir de nouveaux marchés? Ce n'est pas si sûr, car les règles de l'OMC obligent à généraliser les concessions commerciales, ce qui accentue la concurrence entre les PSEM et les Méditerranéens d'outre mer (Chili, Argentine, Australie, Nouvelle Zélande), au détriment des premiers. En décembre 2006, l'UE a accepté de réduire l'obstacle des barrières non tarifaires (un objectif important pour les PSEM vis-à-vis de la PAC), grâce au développement de la convergence réglementaire ${ }^{15}$. L'ouverture doit jouer dans les deux sens, les marchés des pays de la PEV devant aussi s'ouvrir aux exportations communautaires.

42 En principe, l'UE est favorable à la libéralisation des échanges de services, sa balance étant largement excédentaire. Cependant, les difficultés d'application de la soi-disant directive Bolkenstein, qui découlent de l'importance des écarts de revenu dans l'UE élargie se reproduiront quand les PSEM et les pays d'Europe orientale seront en mesure de faire des offres compétitives dans ce secteur. Pour le moment, les progrès sont faibles car les pays du voisinage protègent leur marché, mais il est à craindre que l'UE ne fasse de même quand elle sera menacée.

43 À la différence des précédentes, la libre circulation des capitaux est dans l'intérêt des deux parties et peut donc progresser rapidement. Les propositions de la Commission 
soulignent l'importance de mesures de protection des investissements étrangers, qui pourraient jouer un rôle aussi décisif qu'en Europe centrale, où leur apport représente $5 \%$ du PIB. Mais il revient surtout aux pays du voisinage de prendre les mesures nécessaires (code des investissements, contrôle des changes) pour développer leur attractivité.

Enfin, la Commission est allée encore plus loin en proposant une intégration des économies des pays tiers dans le marché unique, par une mise en œuvre progressive de l'acquis communautaire. Dans les pays de l'EEE, cette expérience a été réalisée dans de bonnes conditions, mais ces pays étaient depuis longtemps en libre-échange avec l'UE pour les produits industriels et avaient des administrations en mesure d'appliquer une législation complexe.

45 L'expérience de l'élargissement de 2004 montre qu'une reprise à marche forcée des 92000 pages de l'acquis communautaire est une entreprise d'une autre nature. Les pays candidats ne s'y sont engagés que parce qu'ils n'avaient pas d'autre choix pour hâter l'adhésion et ils n'y sont pas encore vraiment parvenus. Des carences importantes subsistent et ne seront pas résorbées avant plusieurs années. Car la législation du marché unique a été conçue pour des pays assez ou même très avancés. À l'époque, on n'a pas du tout songé à sa mise en œuvre dans des pays pauvres ou récemment revenus à l'économie de marché. Il faut donc admettre que cet objectif est relativement lointain. Dans les républiques ex-soviétiques qui n'ont pas cette expérience, la capacité administrative serait au moins aussi défaillante qu'en Europe centrale dans les années 1990.

Sur l'ensemble des quatre libertés, on voit que les progrès possibles sont relativement limités, compte tenu des réticences des uns (l'UE avec la libre circulation des personnes) et des incapacités des autres (en matière de libre circulation des services et des capitaux). Seuls les échanges de marchandises sont à même d'augmenter de manière significative, ce qui est déjà possible dans le cadre des accords existants.

Depuis le début des années 1990, l'UE prend lentement conscience des effets territoriaux de ses politiques: impact des fonds structurels, de la PAC, des grands réseaux transeuropéens. Bien que la notion d'espace communautaire soit encore très abstraite, elle progresse à travers de nouveaux concepts comme celui de la cohésion territoriale. Cette dimension prend de l'importance avec le développement de la coopération transfrontalière et transnationale. Une certaine forme de solidarité territoriale émerge à travers la lutte contre la pollution, la sécurité nucléaire ou la définition de nouveaux axes de transport internationaux. Dans cette perspective, le territoire des pays voisins apparait de plus en plus comme solidaire de celui de l'UE.

La coopération dans le secteur des transports fait partie des besoins les plus anciens. La politique des réseaux transeuropéens trouve son prolongement naturel chez les voisins continentaux de l'UE. La modernisation du transport maritime, son développement comme alternative à la congestion, impliquent une coopération avec les PSEM. Comme cela a été fait dans l'UE, un programme d'axes prioritaires euroméditerranéens est à réaliser, après la mise au point du schéma de transport commun et la conférence ministérielle de Marrakech en décembre 2005.

En phase d'accroissement de sa dépendance énergétique, avec la réduction de la production en mer du Nord et son élargissement à l'Europe centrale, l'UE a particulièrement besoin d'une coopération avec ses voisins exportateurs ou pays de transit. La sécurité des approvisionnements passe par le développement d'un réseau 
transcontinental d'oléoducs et de gazoducs et implique une assez grande stabilité des pays concernés. Si l'Europe doit entrer en compétition avec les pays asiatiques (Inde et Chine) pour assurer ses importations, la consolidation de ses relations avec la Russie et les pays de la Caspienne sera un enjeu particulièrement important. C'est dans l'intérêt commun, mais il restera à surmonter des contradictions entre les stratégies nationales, aussi bien dans l'UE qu'entre les pays de la périphérie.

Dans le domaine de l'environnement, voilà longtemps que l'UE a franchi ses frontières en soutenant les efforts des nouveaux membres, de l'Ukraine et de la Russie pour accroître la sécurité nucléaire des centrales électriques et reconvertir les installations militaires. D'une manière plus large, la lutte contre la pollution dans les bassins maritimes et fluviaux a une dimension transnationale évidente: la Méditerranée, la mer Noire, la Baltique, le bassin du Danube ne peuvent être régénérés sans une politique impliquant tous les riverains, notamment ceux qui ne font pas partie de l'UE et qui sont parfois à l'origine des dégâts les plus importants.

51 La coopération territoriale, qui englobe les thèmes ci-dessus, peut se développer dans le cadre géographique des bassins maritimes et fluviaux, où les intérêts communs sont plus visibles : amélioration de l'environnement, organisation d'un réseau de transports, aménagement concerté d'espace fragiles, dans le cadre des nouveaux instruments de coopération, qui interviennent depuis le début de 2007. Ce qui a fonctionné avec un certain succès dans la coopération baltique devrait pouvoir s'étendre à d'autres espaces transnationaux, notamment en Méditerranée. La coopération autour de la mer Noire recevra une attention particulière ${ }^{16}$, ce qui pourrait être un précédent pour créer une organisation transméditerranéenne.

Dans ses propositions de décembre 2006, la Commission prévoit que les pays de la PEV auront accès aux programmes communautaires recherche, éducation (Erasmus) et un appui à la société civile (échanges people to people).

En principe, la nouvelle Politique de voisinage devrait permettre un développement systématique de ces opportunités. Comme un assez grand nombre de ces actions ont été engagées bien avant sa création et que cette politique n'existe que depuis 2004, il n'est pas possible d'en mesurer l'impact spécifique, qui dépend aussi des moyens dont elle aura été dotée.

Dans le cadre des perspectives financières $2007-2013^{17}$, une nouvelle organisation des instruments de l'action extérieure de l'Union a été décidée autour de l'instrument européen de voisinage et de partenariat (IEVP), destiné à succéder, en les fusionnant, aux programmes Tacis (3 milliards d'euros de 2000 à 2006) et Meda (5,35 milliards d'euros de 2000 à 2006), le budget total de 11,2 milliards d'euros couvrant aussi la coopération avec la Russie. La dotation 2007-2013 devrait représenter une allocation moyenne de 4 euros/h et par an pour les 14 pays concernés ${ }^{18}$, ce qui est un peu moins que la dotation Meda 2000-2006 (4,50 euros/h/an) et beaucoup mieux que Tacis (1,50 euro/h/an). $95 \%$ de l'enveloppe ira à des programmes nationaux et $5 \%$ à des coopérations de type Interreg, du côté des pays tiers ${ }^{19}$. Le Feder ${ }^{20}$ financera les interventions sur le côté communautaire, mais les programmes seront gérés par la même direction générale.

Il est clair qu'un budget beaucoup plus important était nécessaire et que la réduction de $20 \%$ opérée par le Conseil par rapport à la proposition de la Commission a donné un mauvais signal. Mais il faut aussi tenir compte des modifications profondes introduites par le règlement dans le processus de gestion. En principe, la reprise de mécanismes 
qui ont fait leurs preuves dans le fonctionnement des fonds structurels (programmation pluriannuelle, cofinancement, coopération avec le Feder pour les programmes de coopération transfrontalière et transnationale) devrait enfin permettre aux pays bénéficiaires de la PEV de consommer intégralement leurs dotations.

Dans ses propositions de décembre 2006, la Commission a ajouté un fonds d'investissement en faveur de la PEV qui pourra servir à mobiliser des fonds supplémentaires de la BEI, avec en perspective, la mise en place d'un système de prêts bonifiés. Enfin, la création d'une facilité " gouvernance » doit compléter les enveloppes nationales dans les pays les plus vertueux, ayant accompli le plus de progrès dans la mise en œuvre de leurs plans d'action.

$\mathrm{Au}$ moment du lancement de la PEV en 2003, les pays souhaitant se placer sur une trajectoire d'adhésion (Géorgie, Ukraine, Moldavie) l'ont perçue comme une opération visant à leur barrer le chemin suivi par les pays d'Europe centrale. Du côté des PSEM, les faibles résultats du processus de Barcelone ne les ont pas incités à lui faire un accueil enthousiaste. Depuis, l'entrée dans la nouvelle période de programmation 2007-2013 et le désir de presque tous les pays de renforcer leurs relations avec l'UE ne laisse pas une grande marge de choix. À Bruxelles comme dans les capitales des pays concernés, on a convenu d'aller le plus loin possible avec cet instrument. À l'exception de la Libye, seul pays méditerranéen (et africain) avec qui l'UE n'a signé aucun accord ${ }^{21}$, et de la Biélorussie (dont l'accord de coopération et de partenariat est suspendu pour déficit démocratique), tous les pays concernés ont accepté de se lancer dans la mise au point des plans d'action.

Les 12 pays $^{22}$ qui les ont réalisés ont suivi une approche à la carte en fonction de leur situation politique. Le Maroc, qui souhaite obtenir de l'UE un statut encore imprécis de partenaire avancé, a cherché à développer systématiquement la coopération dans presque tous les secteurs. En Ukraine, en Moldavie et en Jordanie, le plan d'action occupe une position centrale dans le processus de réforme interne (droits de l'homme, lutte contre la corruption, amélioration de la gestion des finances publiques). En raison de sa situation particulière, l'Autorité palestinienne a retenu les éléments nécessaires à la préparation, puis à la gestion d'un État indépendant. Ailleurs, les plans d'action se concentrent sur les questions commerciales et économiques.

Dans sa configuration initiale, en dépit des ambitions affichées, la PEV ne se présentait pas d'une manière très attractive: elle avait beaucoup de conditionnalités ou de contraintes et peu d'avancées significatives par rapport à ce qui existait déjà par ailleurs ou qui était contenu dans les accords en vigueur. Ensuite, la négociation des plans d'action a montré la difficulté à dépasser le cadre de coopération classique : d'une part, l'offre de l'UE n'est pas jugée assez importante, notamment en ce qui concerne le problème délicat de la circulation des personnes et des budgets de coopération. D'autre part, les pays bénéficiaires n'aiment pas la conditionnalité politique et répugnent à s'engager dans certaines réformes. Il est évident que certains gouvernements (la Biélorussie par exemple) préfèrent de mauvaises relations avec Bruxelles au respect des droits de l'homme qui les obligerait à réduire leur pouvoir.

Car le degré de démocratie reste une pierre d'achoppement. Dans le passé, il n'y a pas eu de réelle mise en œuvre de la conditionnalité politique. Depuis la signature des premiers accords d'association dans les années 1970, l'UE n'a jamais pu faire partager ses aspirations démocratiques aux pays arabes. En dépit des promesses du processus de Barcelone, les PSEM ont été sourds aux recommandations de la Commission et les États 
membres les plus importants préfèrent les dictatures à un processus électoral qui peut conduire au triomphe de l'islamisme radical. En Palestine où les financements communautaires jouaient pourtant un rôle décisif, l'UE n'est pas parvenue à imposer un progrès des libertés, ni même une gestion transparente des aides. Sauf au Liban, les forces démocratiques n'ont pas reçu un soutien comparable à celui offert en Europe centrale, ou même dans certains pays africains.

61 Cependant, la Commission a réitéré ses exigences en matière de meilleure gouvernance, confirmant l'importance des critères politiques. Les entorses à la démocratie ne sont plus acceptables, mais cela est plus facile à dire à Minsk qu'à Moscou. À la suspension des relations avec la Biélorussie, où les violations des droits de l'homme sont particulièrement nombreuses, fait pendant une approche plus pragmatique avec d'autres pays, en dépit des libertés prises avec les droits de l'homme en Égypte, en Tunisie ou même en Russie.

62 À vrai dire, les exigences politiques n'ont été pleinement acceptées et n'ont joué un rôle déterminant qu'en Europe centrale, parce qu'elles conditionnaient l'adhésion. Sans cette perspective, elles sont moins efficaces. La mise en œuvre des clauses de suspension est hautement politique et les États membres n'ont pas vocation à suivre automatiquement les recommandations de la Commission. La marge d'appréciation est variable et pourrait rester assez large aussi longtemps que la Russie continue à donner le mauvais exemple.

63 Comme ailleurs, dans le « débat opposant idéalistes wilsoniens d'une part et tenants de la Realpolitik de l'autre» ${ }^{23}$, la politique étrangère de l'Union ne peut aller dans un seul sens. Il est possible que l'UE renonce à une partie de ses exigences pour éviter des problèmes à ses frontières :

64 - la lutte contre le terrorisme et la criminalité transnationale, éventuellement l'immigration clandestine, conduisent à privilégier l'efficacité, donc à coopérer avec des régimes non démocratiques et à leur donner des gages, ce que font les États-Unis depuis le 11 septembre 2001, tout en gardant un discours ferme sur les principes ;

65 - la dépendance énergétique de l'UE s'est accrue avec l'élargissement et les pays qui contrôlent les ressources d'hydrocarbures sont presque tous non démocratiques. Il est donc clair que la sécurité des approvisionnements et l'État de droit sont plus aisément compatibles sur le long terme que dans l'immédiat, notamment quand le prix des hydrocarbures est en forte hausse.

66 Avec les pays candidats, notamment la Croatie et la Turquie, on a utilisé la conditionnalité négative: ne pas ouvrir les négociations d'adhésion, ou bloquer un chapitre de celle-ci, aussi longtemps que certains résultats n'avaient pas été atteints. Faut-il instituer dans la Politique de voisinage une conditionnalité positive ? Faute de pouvoir lancer des mesures punitives, qui auraient des effets non souhaités, y compris pour l'UE, il s'agirait d'encourager la démocratie en aidant les pays qui sont sur cette voie à progresser en liant une partie des aides à des résultats démocratiques.

67 Enfin, parmi les pays qui forment le grand voisinage de l'Europe ${ }^{24}$, les archipels de l'océan indien et de l'Atlantique aimeraient voir leurs mérites, qu'ils estiment au moins équivalents à ceux de la majorité des pays de la $\mathrm{PEV}$, pris en considération. Bien classés par l'indice de développement humain du PNUD, reconnus comme pleinement démocratiques ${ }^{25}$, ils voudraient aller plus loin dans leur coopération avec l'Union, que le cadre ACP auquel ils appartiennent actuellement. C'est le cas de l'archipel du Cap 
Vert, qui s'est engagé dans la mise au point d'un partenariat spécial. S'il aboutit, celuici devrait être très proche du modèle de la politique européenne de voisinage.

\section{NOTES}

1. "L'adhésion requiert du pays candidat qu'il ait des institutions stables, garantissant la démocratie, la primauté du droit, les droits de l'homme, le respect des minorités et leur protection...»

2. Dont l'article $6 \$ 1$ stipule que «l'Union est fondée sur les principes de la liberté, de la démocratie, du respect des droits de l'homme et des libertés fondamentales ainsi que de l'État de droit, principes qui sont communs aux États membres. »

3. Agenda 2000, tome 1, p. 67.

4. Voir dans Futuribles, $\mathrm{n}^{\circ}$ 331, juin 2007, «Union européenne : la résistance turque », p. 37-48.

5. Mais ni les quatre TOM (Nouvelle Calédonie, Polynésie, Wallis et Futuna, Terres australes et antarctiques), ni Mayotte et Saint Pierre et Miquelon.

6. Les villes de Ceuta et Melilla et quelques petites îles.

7. Une douzaine de confettis de l'empire, dispersés entre les Antilles et les trois grands océans.

8. Ensemble formé par les îles de l'Atlantique à l'ouest de l'Afrique : Açores, Madère, Canaries et archipel du Cap Vert.

9. Communauté des États indépendants, association faiblement structurée qui regroupe les Républiques ex-soviétiques, à l'exception des Baltes.

10. Les 10 PSEM (Maroc, Algérie, Tunisie, Libye, Égypte, Jordanie, Syrie, Liban, Israël et l'Autorité palestinienne) et les six républiques ex-soviétiques européennes (Biélorussie, Ukraine, Moldavie, Géorgie, Arménie et Azerbaïdjan).

11. Meda : programme européen de coopération avec les PSEM.

12. Tacis : Technical Assistance to the Community of Independent States : programme européen de coopération avec la Mongolie et les républiques ex-soviétiques (à l'exception des Baltes).

13. Liberté de circulation des personnes, des marchandises, des prestations de service et des capitaux.

14. La règle de l'unanimité la met dans une posture défensive.

15. Ce qui revient à dire aux pays tiers : vos produits entreront facilement dans l'UE si vous appliquez notre réglementation.

16. La Commission soutiendra la BSEC (Organization of the Black Sea Economic Cooperation).

17. Adoption du règlement $1638 / 2006$ établissant un instrument de voisinage et de partenariat le 24 octobre 2006.

18. La coopération avec la Biélorussie est suspendue et la Libye n'a pas signé d'accord.

19. Soit une moyenne de $80 \mathrm{M}$ e/an (le double de ce qui a été réservé en 2004-2006 au titre de l'instrument de voisinage, 75M pour TACIS et 45M pour MEDA, soit 40M e/an).

20. Fonds européen de développement régional.

21. Dans un mémorandum signé entre la Commission et le gouvernement libyen le 23 juillet 2007 à l'occasion de la libération des infirmières et du médecin bulgares, il est fait référence à un accord spécifique que la Commission s'engage à proposer au Conseil, couvrant cinq thèmes de coopération, dont l'ouverture du marché européen aux exportations libyennes, une aide 
européenne à l'archéologie et la mise en place d'un dispositif commun de lutte contre l'immigration clandestine.

22. L'Algérie et la Syrie sont en retard.

23. Madeleine Albright, 2003, Madame le Secrétaire d'État, Mémoires, Paris, Albin Michel.

24. Selon le terme utilisé par les textes communautaires qui traitent de l'environnement géographique des régions ultrapériphériques (RUP).

25. L'Ibrahim Index of African Governance, 7/04/2008.

\section{RÉSUMÉS}

Depuis le $1^{\text {er }}$ janvier 2007, l'Union européenne compte 27 États. En moins de deux décennies, elle a plus que doublé le nombre de ses membres, mais il semble que son élargissement ne soit pas achevé. À la Croatie et à la Turquie déjà engagées dans des négociations d'adhésion; la Macédoine a le statut de candidat officiel; les autres pays des Balkans occidentaux sont liés à l'Union par des accords explicitement conçus pour les amener à l'adhésion; certains pays de la périphérie orientale sont candidats à la candidature et attendent une occasion favorable pour déposer officiellement leur demande (Ukraine, Moldavie, Géorgie). Qu'ils s'inscrivent ou non dans une perspective d'adhésion, tous ces pays sont engagés dans un mouvement de recomposition qui n'a qu'un mot d'ordre: «no western borders». Une vingtaine de peuples se rapprochent du seul ensemble qui puisse leur offrir, soit une perspective d'intégration, soit un processus de rapprochement graduel dans le cadre de la nouvelle Politique européenne de voisinage (PEV). Face à la rapidité de ce processus, on ne peut s'empêcher de se poser quelques questions redoutables. Est-il possible de maîtriser cette évolution, parfois qualifiée de scénario des dominos, dont les limites ne sont pas définies a priori? La politique de voisinage, mise en œuvre en 2003, permet-elle de dépasser le problème d'un élargissement sans fin dans un scénario des dominos incontrôlé?

Since the beginning of 2007, the European Union is composed by 27 countries. In less than twenty years, the number of the member states has more than doubled by it seems that its enlargement has not come to an end yet. Croatia and Turkey have already started the negotiation towards a full membership status. The FYROM is an official candidate. The other countries of Western Balkans are linked to the EU by association agreements clearly conceived to lead them to the accession. Some countries of the eastern neighbourhood have officially expressed their will to become members of the European Union and they are just waiting to right moment to apply for an official candidate status (Ukraine, Moldova, Georgia). Whether they are in a perspective of accession or not, all these countries are engaged in a process of restructuration based on one official motto : «no western borders ». Almost twenty European people are step by step getting closer to one alternative : either the perspective of the accession, or a process of gradual mutual rapprochement in the framework of the new European Neighbourhood Policy. Some important questions are raised by the rapidity of these evolutions. Is it possible to handle this so-called "dominos scenario" whose final limits have never been determined? Is the implementation of the neighbourhood policy a way to avoid the scenario of an endless and uncontrolled enlargement? 
INDEX

Mots-clés : Union européenne, intégration européenne, scénario des dominos, voisinage

Index géographique : Europe, Croatie, Turquie, Ukraine, Moldavie, Géorgie, Macédoine, Balkans

\section{AUTEUR}

JEAN-FRANÇOIS DREVET

Ancien fonctionnaire à la Commission européenne 